\title{
Experimental investigation of frequency characteristics of the multistage slot membranes waveguide filters
}

\author{
Natalia Kopylova ${ }^{1, *}$, Alexei Kopylov ${ }^{1}$, and Yuri Salomatov ${ }^{1}$ \\ ${ }^{1}$ Siberian Federal University, 660079 Krasnoyarsk, Russia
}

\begin{abstract}
In this article, we present results of experimental investigation of amplitude-frequency characteristics (AFC) of the five-stage "filters with the waveguide-slot membranes" (FWSM) in the range of frequencies $5.4 \ldots 8.5 \mathrm{GHz}$ for six filters. Article shows the following trend change filter frequency response by increasing the width of the slots of the second and fourth membranes: decrease the quality factor, resonant frequency increase, reducing losses in the passband.
\end{abstract}

\section{Introduction}

There are a lot of papers of waveguide filters with metal membranes, which are arranged perpendicular to the direction of propagation of electromagnetic waves [1-5]. These works cover a large historical period: from 1946 [1] to 2000-ies [2-5]. These filters are called «iris filters» ([3-5]). However, it is more convenient to name them "filters with the waveguideslot membranes" (FWSM). Using filters of this type allows getting wide bandwidth or attenuation with low weight and sizing characteristics of the filter. Taking such filters is the most important in the centimeter wavelength range of the microwave to minimize their size in comparison with conventional filters on long line segments.

The results of experimental studies of amplitude-frequency characteristics (AFC) of one of these types of filters in the frequency range $5.4 \ldots 8.5 \mathrm{GHz}$ we present in this article.

\section{Filters design}

The structure of the investigated five-stage FWSM is schematically shown in the Figure 1. Figure 1a) shows generalized filters topology, Figure 1b) - filters on the side.

Our five-stage FWSM contains a set of five waveguide-slot membranes with " $B$ " wide of slot (Figure 1a)). The value "B" is constant for the first, the third and the fifth membranes. For the first and for the fifth membranes, it is equal to $22 \mathrm{~mm}$ and for the third membrane- $15 \mathrm{~mm}$. For the second and for the fourth membranes value "B" is $1 \mathrm{~mm}$, or 2 $\mathrm{mm}$, or $3 \mathrm{~mm}$, or $4 \mathrm{~mm}$, or $5 \mathrm{~mm}$, or $6 \mathrm{~mm}$. Slot is arranged parallel to broad wall of the waveguide. The cross section of the waveguide is $35 \times 15 \mathrm{~mm}$. The thicknesses of the filter

*Corresponding author: kopaph@yandex.ru 
membranes are: $6 \mathrm{~mm}$ for $\mathrm{S} 1$ and $\mathrm{S} 5 ; 10 \mathrm{~mm}$ for S3; $0.25 \mathrm{~mm}$ for S2 and $\mathrm{S} 4$ respectively (Figure 1b)).

Thus, the design of the FWSM is a symmetrical structure consisting of five cascade waveguide-slot membranes with different membrane thicknesses and slots width. The lengths of the slots of the membranes are chosen equal to the length of the broad wall of the waveguide. All slot membranes disposed along to the common (based) side of the waveguide (Figure 1b) with a bold type cross-hatching line).
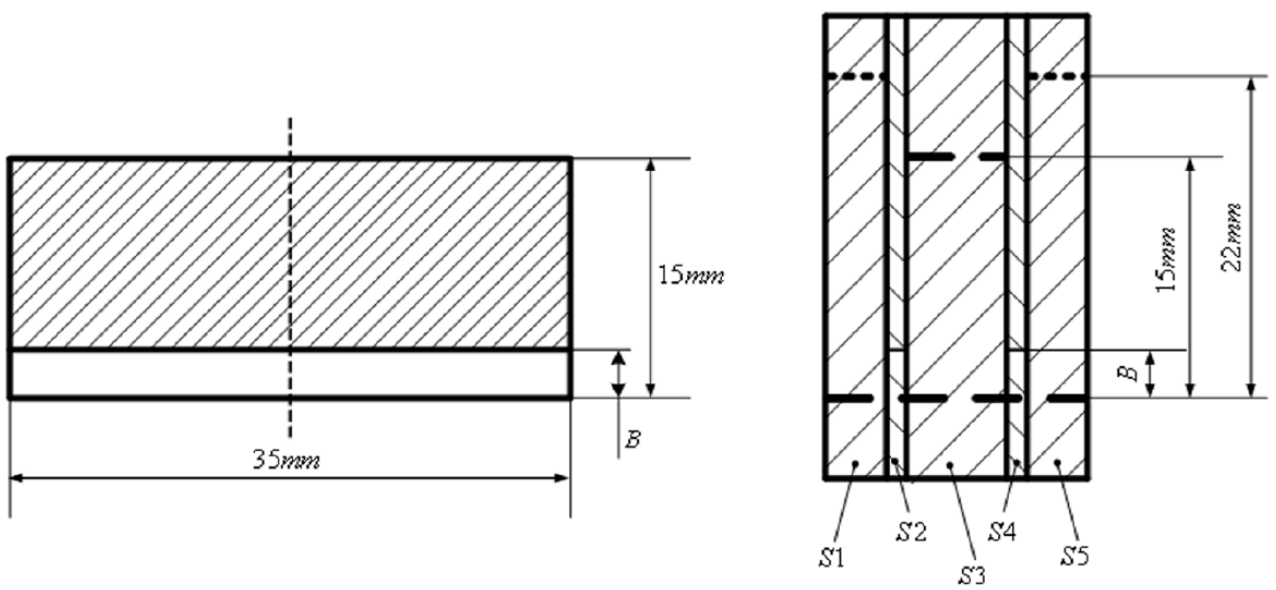

Fig. 1. The structure of the investigated five-stage "filter with waveguide-slotted membrane" (FWSM): a) Generalized filters topology, b) Filters on the side.

The filter design was selected by us experimentally to determine the form of the amplitude-frequency characteristics of the filter in bandwidth and influence of the width of slots of waveguide-slot membranes.

\section{Results and discussions}

For our investigations, we made six FWSM with structure shown in Figure 1 and different wide of "B" slot. The value of "B" was for the first filter equal to $1 \mathrm{~mm}$, for the second filter $-2 \mathrm{~mm}$, for the third filter $-3 \mathrm{~mm}$, for the fourth filter $-4 \mathrm{~mm}$, for the fifth filter -5 $\mathrm{mm}$, and for the sixth filter $-6 \mathrm{~mm}$.

Figure 2 demonstrates amplitude-frequency characteristics (AFC) of all six filters investigated by us. Figure 2a) shows the AFC of the first FWSM which value of "B" is 1 $\mathrm{mm}$; Figure $2 \mathrm{~b}$ ) - the second FWSM which value of " $\mathrm{B}$ " is $2 \mathrm{~mm}$; Figure $2 \mathrm{c}$ ) - the third FWSM which value of " $B$ " is $3 \mathrm{~mm}$; Figure $2 d$ ) - the fourth FWSM which value of " $B$ " is $4 \mathrm{~mm}$; Figure 2e) - the fifth FWSM which value of " $\mathrm{B}$ " is $5 \mathrm{~mm}$; and Figure $2 \mathrm{f}$ ) - the sixth FWSM which value of " $\mathrm{B}$ " is $6 \mathrm{~mm}$.

Course of frequency dependencies of transmission filters' coefficient in Figure 2 proves: investigated filters allow to implement filters of AFC type low-pass filter (LPF) (Figure 2a)); filters with AFC type of band-pass (BPF) filter in the Figure 2b), 2c), 2d), 2e) and AFC-type high-pass filter (HPF) in the Figure 2f). Moreover, our FWSM's exhibit the properties of band-stop filter. This is observed at frequencies above $7 \mathrm{GHz}$ in filters, which frequency responses are shown in Figure 2a), 2b), 2c), and in filter with frequency response is shown in Figure 2f) at frequencies less than $6 \mathrm{GHz}$. 


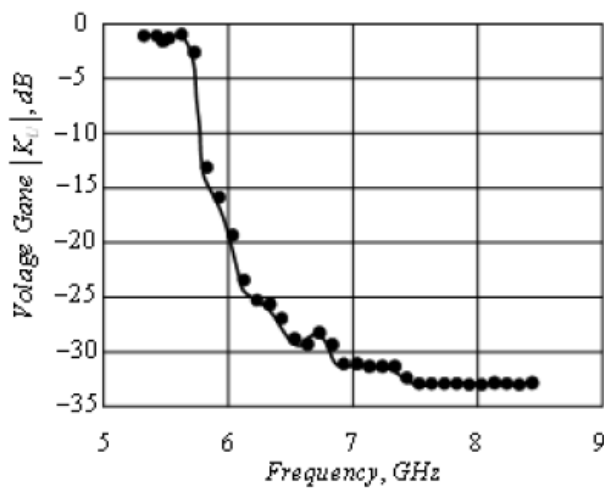

a)

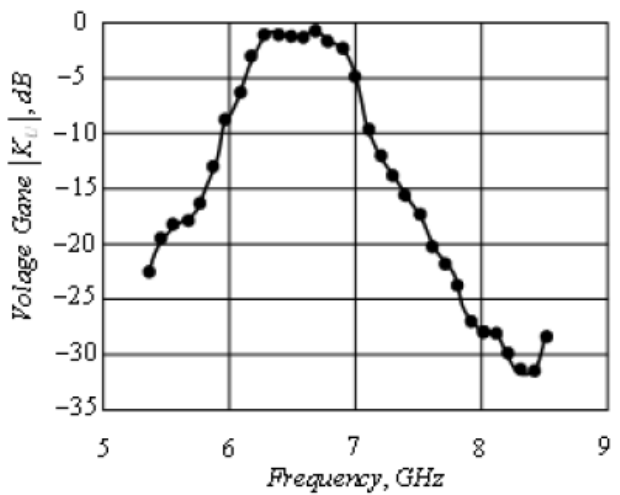

c)

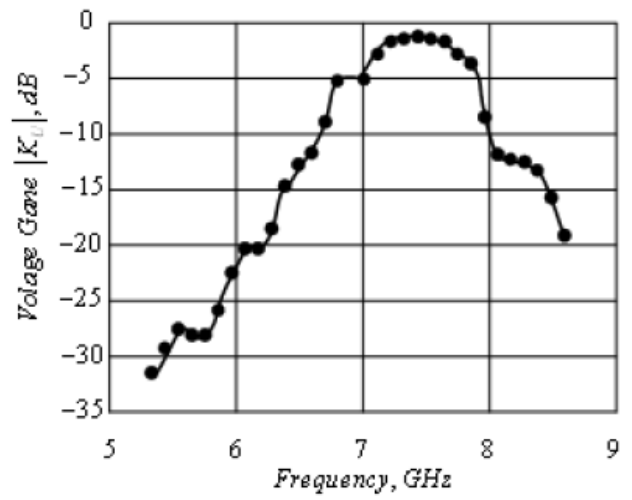

e)

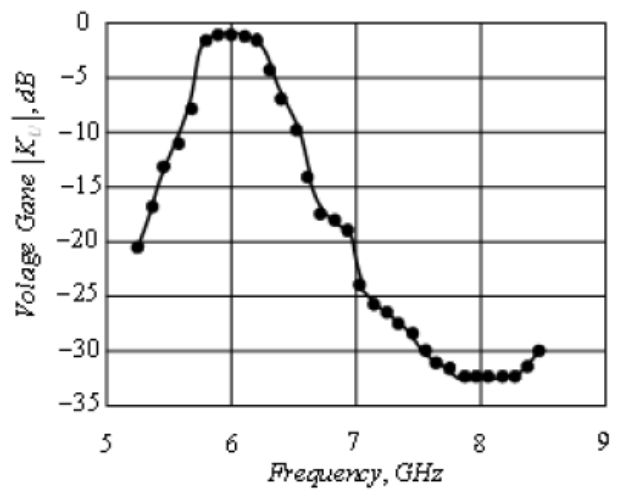

b)

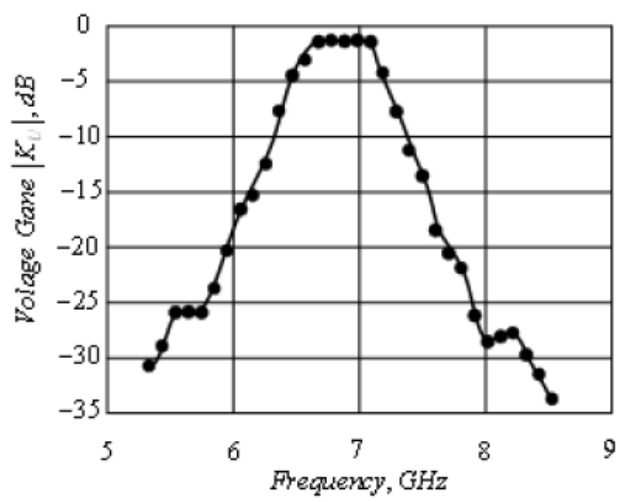

d)

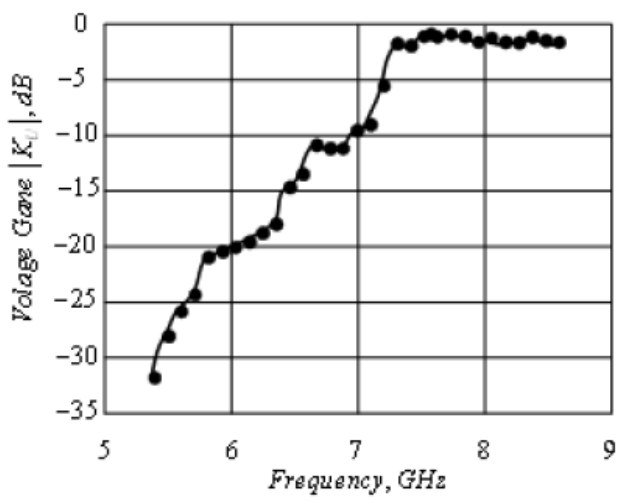

f)

Fig. 2. Amplitude-frequency characteristics of all six filters investigated by us: 2 a) AFC of the first FWSM; 2b) AFC of the second FWSM; 2c) AFC of the third FWSM; 2d) AFC of the fourth FWSM; 2e) AFC of the fifth FWSM; 2f) AFC of the sixth FWSM.

Besides, the band-pass has been expanding with increasing "B" magnitude of gap in the second and fourth filter membranes. At the same time we notice decreasing slope of the 
filters' frequency characteristics. Both of going facts are easily explained by increasing of bond between filter elements with increasing the "B" value of second and fourth membranes' gaps. We notice the values of insertion loss in the bandwidth for all investigated filter is up to $0.1 \mathrm{~dB}$, which is the limit of the measurements' accuracy as well.

In addition, we should notice a significant change in the minimum attenuation of AFC filters on frequencies: changing of the "B" amount from $1 \mathrm{~mm}$ to $6 \mathrm{~mm}$ in the second and fourth filters membranes comes to changes of these frequencies' value from $5.5 \mathrm{GHz}$ to 7.5 GHz.

\section{Conclusions}

We believe these results are useful for realization of waveguide filters with different frequency response types for some areas such as radio communication, radio navigation and radar equipment of wide applications.

\section{References}

1. R.M. Fano, A.W. Louson, RLE, Mass. Inst. Techn. 8, 1 (1946)

2. J. Carbonell, J. Roglá Luis, V. E. Boria, D. Lippens, IEEE Trans. 4, 1527 (2006)

3. I. Stevanovic', P. Crespo-Valero, R. J. Mosig, IEEE Trans. 1, 189 (2006)

4. Q. F. Zhang, Y. L. Lu, IET Microw. Ant. Prop. 4, 1256 (2010)

5. C. A. Leal-Sevillano, J. R. Montejo-Garai, J., A. Ruiz-Cruz, J. M. Rebollar, IEEE Trans. 3, 1 (2016) 